\title{
Persistent Eosinophilic Inflammation in Adult Asthmatics with High Serum and Urine Levels of Leukotriene $\mathrm{E}_{4}$
}

\author{
Ga-Young Ban $\mathbb{1}^{1,2}$ \\ Seung-Hyun Kim ${ }^{3}$ \\ Hae-Sim Park (iD) ${ }^{4}$ \\ 'Department of Pulmonary, Allergy and \\ Critical Care Medicine, Kangdong Sacred \\ Heart Hospital, Hallym University \\ College of Medicine, Seoul, Korea; \\ ${ }^{2}$ Allergy and Clinical Immunology \\ Research Center, Hallym University \\ College of Medicine, Chuncheon, Korea; \\ ${ }^{3}$ Translational Research Laboratory for \\ Inflammatory Disease, Clinical Trial \\ Center, Ajou University Medical Center, \\ Suwon, 16499, Korea; ${ }^{4}$ Department of \\ Allergy and Clinical Immunology, Ajou \\ University School of Medicine, Suwon, \\ Korea
}

Background: Cysteinyl leukotrienes (CysLTs) are key mediators for bronchoconstriction, eosinophil recruitment and mucus production in the airways of asthmatic patients. To better understand the role of CysLTs in different asthma phenotypes, we compared the levels of arachidonic acid metabolites in relation to asthma control status and phenotypes in adult asthmatics on regular anti-asthma medications.

Methods: A total of 137 adult asthmatics (47 with aspirin-exacerbated respiratory disease [AERD] and 90 asthmatics with aspirin-tolerant asthma [ATA]) and 20 healthy controls were enrolled. Arachidonic acid metabolites in serum and urine were analyzed using LC-MS/MS methods, and clinical data, including asthma control status, exhaled NO (FeNO) and lung function tests, were collected.

Results: Urine $\mathrm{LTE}_{4}$ levels were significantly higher in AERD patients on inhaled corticosteroid-long-acting $\beta_{2}$ - agonist plus leukotriene receptor antagonist (LTRA) treatment than in ATA patients $(P=0.001)$. No differences were found in the serum or urine levels of 15 -HETE, $\mathrm{TXB}_{2}$, or $\mathrm{PGF}_{2 \alpha}$. High serum $\mathrm{LTE}_{4}$ levels were associated with lower FEV1\% and uncontrolled status in AERD patients ( $P=0.006$ and $P=0.002$, respectively), but not in ATA patients. Multivariate analysis demonstrated that blood eosinophil counts, FeNO levels and aspirin hypersensitivity were significant factors affecting urine $\mathrm{LTE}_{4}$ levels.

Conclusion: Despite LTRA treatment in AERD, the $\mathrm{LTE}_{4}$ levels remained high and showed close associations with blood eosinophilia, high FeNO levels and impaired disease control. Our real-world evidence indicates that control of asthma is not fully achieved by blocking the CysLT pathway with LTRA. Thus, introduction of treatment modalities targeting eosinophilia could be a better option for patients with high CysLTs.

Keywords: asthma, leukotrienes, asthma control, aspirin-exacerbated respiratory disease, eosinophil

\section{Introduction}

Asthma is a heterogeneous disease with many clinical phenotypes characterized by variable airflow obstruction, chronic airway inflammation and bronchial hyperresponsiveness. Asthma is usually controlled by the regular use of an inhaled corticosteroid (ICS) with or without a long-acting $\beta_{2}$-agonist (LABA) or a leukotriene receptor antagonist (LTRA). Despite the standard asthma treatment, about $10 \%$ to $20 \%$ of patients still remain uncontrolled, often with frequent exacerbations. ${ }^{1,2}$

The pathophysiology of asthma is complex, and multicellular inflammatory processes including eosinophils, neutrophils, $\mathrm{T}$ helper cells, monocytes, mast
Correspondence: Hae-Sim Park Department of Allergy and Clinical Immunology, Ajou University School of Medicine, Worldcup-ro 164, Youngtong-gu, Suwon-si, Suwon, 443-380, Korea

Tel +82-3I-2I9-5I50

Fax +82-31-219-5I54

Email hspark@ajou.ac.kr 
cells, basophils and epithelial cells with various chemical mediators and cytokines are involved. ${ }^{3}$ Cysteinyl leukotrienes (CysLTs), namely $\mathrm{LTC}_{4}, \mathrm{LTD}_{4}$ and $\mathrm{LTE}_{4}$, have been suggested to be important inflammatory mediators enhancing airway inflammation and uncontrolled asthma, especially for patients with aspirin-exacerbated respiratory disease (AERD). They are produced in a cascade manner via the arachidonic acid (AA) pathway by mast cells and eosinophils in the airways. ${ }^{4}$

$\mathrm{LTE}_{4}$, a stable and reliable metabolite, has been detected in serum and urine, and used as an indicator for the activation of AA or CysLT pathways. ${ }^{5}$ Higher urine levels of $\mathrm{LTE}_{4}\left(\mathrm{uLTE}_{4}\right)$ have been reported in patients with moderate to severe asthma as well as with AERD, in whom activation of the CysLT pathway is related to eosinophil recruitment into the airways, ${ }^{6}$ and shows a negative correlation with $\mathrm{FEV}_{1}{ }^{7}$ Leukotriene receptor antagonists (LTRAs), including montelukast, zafirlukast and pranlukast, have been developed to reduce CysLTs-mediated airway inflammation in asthmatics. ${ }^{8}$ CysLTs are known to cause bronchoconstriction, mucosal edema and mucus secretion. ${ }^{9}$ Moreover, they are involved in airway remodeling via activating epithelial cells. Previous studies have revealed the role of CysLTs in airway remodeling via TGF- $\beta 1$ production ${ }^{10}$ and enhancing TGF- $\beta 1$ response. ${ }^{11}$ The clinical efficacy of LTRAs in adult asthmatics has been reported in some studies, in which addition of LTRAs improves asthma control status in adult asthmatics whose symptoms remain uncontrolled despite the use of ICSs. ${ }^{12}$ However, some asthmatics do not respond adequately to LTRAs, even together with ICS and LABA, and the detection of high $\mathrm{LTE}_{4}$ levels in urine or serum has been suggested to enrich the responders. To date, there have been few studies to examine whether airway inflammatory responses remain uncontrolled with use of LTRAs in adult patients with AERD or aspirin-tolerant asthma (ATA).

The primary objective of the present study was to examine the levels of AA metabolites according to LTRA medication as well as anti-inflammatory medication including ICS, and to find whether the LTRA treatment could suppress the levels of $\mathrm{LTE}_{4}$. Furthermore, we evaluated whether the levels of AA metabolites are associated with asthma control status and the degree of eosinophilic inflammation in a realworld clinical setting involving patients with AERD and those with ATA.

\section{Methods}

\section{Study Design and Study Subjects}

This study was a prospective cross-sectional and noninterventional real-world study. We enrolled 47 patients with AERD, 90 patients with ATA and 20 normal healthy controls (HC) at Ajou University Hospital in Suwon, South Korea. Asthma was diagnosed according to the GINA by the allergy specialists. Exclusion criteria for enrollment were as follows: 1) asthmatics who had ever been treated with type 2 biologics, including omalizumab, mepolizumab, reslizumab and dupilumab, within 130 days of enrollment, 2) current smokers or ex-smokers who quit smoking within 30 days of enrollment; and 3) asthmatics whose controller medications were changed within 7 days of enrollment.

Study subjects were recruited according to current maintenance medications, especially LTRA as well as ICS-LABA. In addition, they were grouped by NSAID/ aspirin hypersensitivity because the levels of AA metabolites are known to be different in patients with AERD compared to those with ATA. ${ }^{5}$ The ATA group was divided into 2 subgroups: ATA with ICS-LABA and ATA with ICS-LABA plus LTRA. In the AERD group, all the AERD patients enrolled had been taking ICS-LABA plus LTRA (Figure 1). The doses of ICS were grouped into low, medium and high doses as defined in the Global Initiative for Asthma guideline (GINA) guideline. ${ }^{13}$

AERD was defined by a typical clinical history (recurrent exacerbation of upper or lower respiratory reactions after ingestion of aspirin/NSAIDs) and/or a positive response to the lysine-aspirin bronchial provocation test (Lys-ASA BPT). ${ }^{14}$ The Lys-ASA BPT was performed with increasing doses of Lys-ASA solution up to $300 \mathrm{mg} / \mathrm{mL}$ using the method previously reported. ${ }^{15}$ The result of the Lys-ASA BPT was considered "positive" if $\mathrm{FEV}_{1} \%$ was decreased by more than $20 \%$ after the challenge. Subjects who showed negative results to the Lys-ASA BPT or denied any changes in upper or lower respiratory tract symptoms after ingestion of aspirin/NSAIDs were defined as ATA.

To evaluate the levels of AA metabolites according to asthma control status, study subjects were grouped by asthma control status (well controlled, partly controlled or uncontrolled status) based on the GINA guideline. Wellcontrolled asthma was defined if patients experienced daytime symptoms and reliever use less than twice a week without night awakening or activity limitation in the past 4 


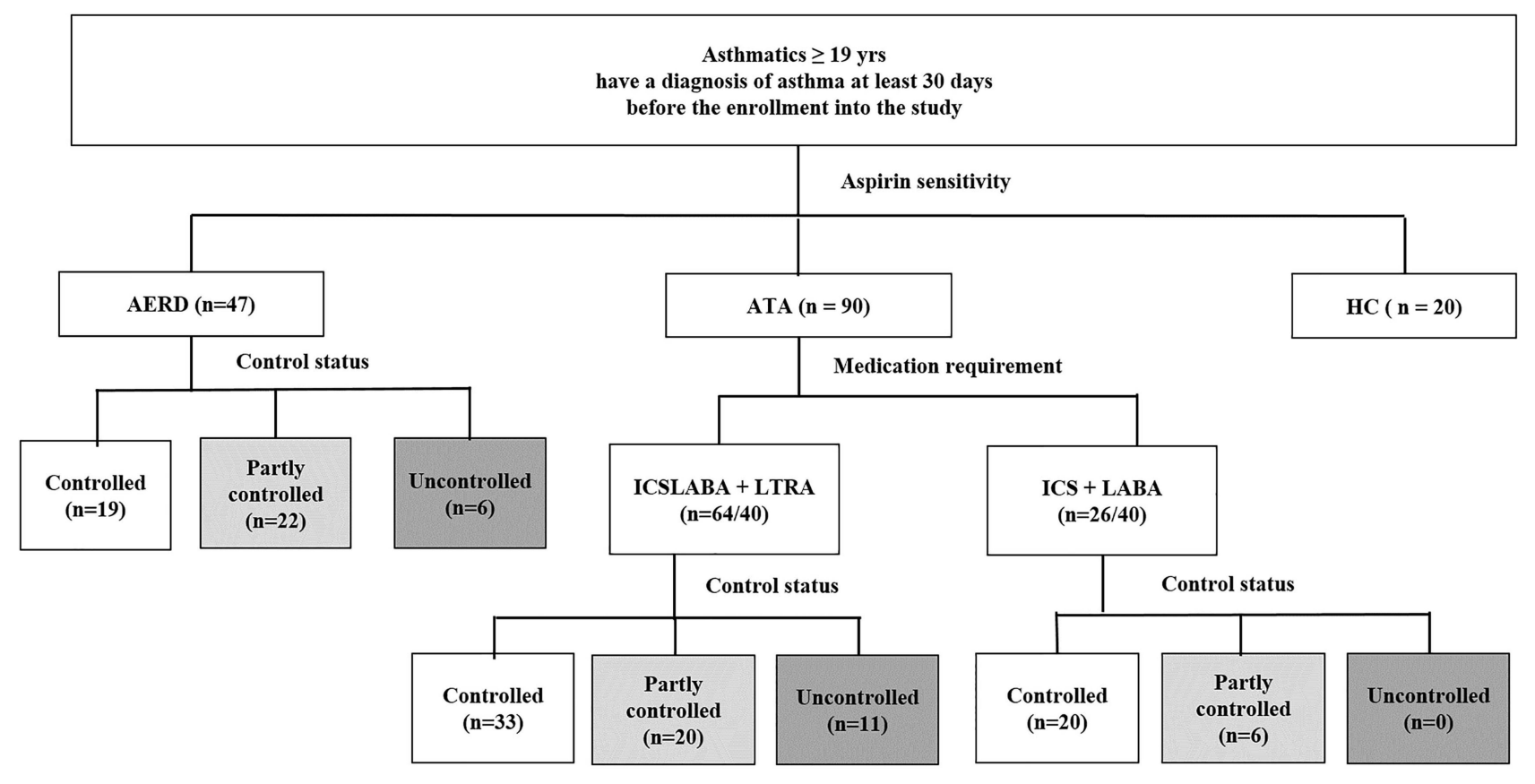

Figure I Study subject group enrolled in the present study.

Note: Asthma control status was defined by the GINA guideline.

Abbreviations: AERD, aspirin-exacerbated respiratory disease; ATA, aspirin tolerant asthma; HC, healthy control; ICSLABA, inhaled corticosteroid/long-acting betaagonist; LTRA, leukotriene receptor antagonist.

weeks. Uncontrolled asthma was defined if asthmatics showed ACT $\leq 20^{8,16}$ or ACQ- $6 \geq 1.5 .{ }^{17}$ Severe asthma was defined according to the American Thoracic Society Workshop. ${ }^{18}$ All subjects gave written informed consent at the time of enrolment, and the study was approved by the Institutional Review Board of Ajou University Hospital (AJIRB-BMR-SUR-15-498). This study was conducted in accordance with the Declaration of Helsinki.

\section{Collection of Clinical Data and Samples}

Peripheral venous blood, sputum and urine samples were collected from the subjects between 8:00 AM and 11:00 $\mathrm{AM}$ when the patients were maintained on controller medications including ICS-LABA and LTRAs. Blood eosinophil counts, sputum eosinophil counts and total immunoglobulin E (IgE) levels were analyzed. The serum and urine levels of metabolites, including $\mathrm{LTE}_{4}, 15-$ hydroxyeicosatetraenoic acid (15-HETE), 11-dehydro thromboxane $\mathrm{B}_{2}\left(\mathrm{TXB}_{2}\right)$ and prostaglandin $\mathrm{F}_{2 \alpha}\left(\mathrm{PGF}_{2 \alpha}\right)$, were quantified using liquid chromatography-tandem mass spectrometry (LC-MS/MS). LTE L $_{4} \mathrm{~d}_{5}$, 15(S)-HETE-d 8 , $\mathrm{TXB}_{2}-\mathrm{d}_{4}$ and 8-iso $\mathrm{PGF}_{2 \alpha}-\mathrm{d}_{4}$ were used as internal standards. Chromatographic separation was performed using a Waters Acquity UPLC system (Waters) with a Hypersil GOLD column $(2.1 \times 100 \mathrm{~mm}, 1.9 \mu \mathrm{m}$ : ThermoFisher
Scientific, San Jose, CA, USA). Data were obtained using an API5500 triple quadrupole mass spectrometer (AB Sciex, Framingham, MA, USA) equipped with an ESI source. For the quantitative determination of creatinine in urine samples, $10 \mu \mathrm{L}$ of the urine sample were applied to the Creatinine Parameter Assay Kit (R\&D Systems, Minneapolis, MN, USA). ${ }^{19}$ Pulmonary function test, fractional exhaled nitric oxide (FeNO) measurement, questionnaires survey using the asthma control test (ACT), and asthma quality of life (AQLQ) and asthma control questionnaire (ACQ-6: ACQ mean of 6 individual item scores) were performed on the same day of enrollment.

\section{Measurement of Serum and Urine Levels of Metabolites}

Serum and urine samples stored at $-70^{\circ} \mathrm{C}$ were thawed and analyzed by LC-MS/MS. Chromatographic separations of metabolites in urine and serum were performed with a Hypersil GOLD $(2.1 \quad \mathrm{X} \quad 100 \quad \mathrm{~mm}, \quad 1.9 \mu \mathrm{m}$ : ThermoScientific, San Jose, CA, USA). The eluent was introduced into an AB Sciex, API5500 Triple Quadrupole mass spectrometer. The following deuterated internal standards were used: $\mathrm{LTE}_{4}$-d5 for $\mathrm{LTE}_{4}, 15(\mathrm{~S})$-HETE-d8 for 15-HETE, 11-dehydro $\mathrm{TXB}_{2}$-d4 for 11-dehydro $\mathrm{TXB}_{2}$ and 8-iso $\mathrm{PGF}_{2} \alpha$-d4 for $\mathrm{PGF}_{2 \alpha}$ (Cayman Chemical Company, 
Ann Arbor, MI, USA). In urine samples, creatinine was also quantified to normalize to the actual concentrations of each biomarker and 1,3-dimethyl-2-imidazolidinone (Sigma-Aldrich, St. Louis, MO, USA) and then used as the internal standard for creatinine.

\section{Statistical Analysis}

Continuous variables were compared using Student's $t$-test, and Pearson's chi-squared or Fisher's exact test were used for categorical variables. Analysis of variance was performed for comparisons among the 3 groups. General linear regression analysis was performed to adjust for the confounding factors age and sex, and to compare metabolite levels between the groups. Pearson's correlation analysis identified associations among continuous variables. All computations were performed using SPSS software, version 22.0 (IBM Corp., Armonk, NY, USA). GraphPad Prism 5.0 software (GraphPad Inc., San Diego, CA, USA) was used for the production of graphs.

\section{Results}

\section{Clinical Characteristics and Asthma Control Status of the Study Subjects}

Table 1 summarizes the demographic information of all the study subjects on the day of enrollment. ICSs that were prescribed to the study subjects were beclomethasone, budesonide and fluticasone. The proportion of low-, medium or high-dose ICS users was not significantly different between patients with AERD and ATA (low-dose: 25.5\% vs $15.6 \%$, medium-dose: $57.4 \%$ vs $59.4 \%$, high-dose: $17.0 \%$ vs $25.0 \% ; P>0.05$ for all). LTRAs that were used in the study subjects were montelukast $(10 \mathrm{mg} /$ day) and pranlukast (450mg/day). The AERD group showed higher blood eosinophil counts but lower levels of total $\mathrm{IgE}$ compared to the ATA group $(P=0.011$ and $P=0.029$, respectively), while no significant differences were observed in lung function parameters. There were no significant differences in asthma control status between the AERD and ATA groups. The ACT, ACQ or AQLQ scores showed no significant differences between the AERD and ATA groups (ACT:21.15 \pm 3.11 vs $20.40 \pm 4.06$, ACQ: $4.60 \pm 4.95$ vs $6.32 \pm 5.74$, AQLQ: $172.72 \pm 37.04$ vs $165.82 \pm 38.84 ; P>0.05$ for all). Inflammatory parameters or lung functions showed no significant differences between ATA patients with ICS/LABA and with ICS/ LABA plus LTRA. However, a higher proportion of wellcontrolled asthmatics, as defined by the GINA guideline, was observed in ATA patients with ICS/LABA than with ICS/LABA plus LTRA $(P=0.031)$. The ACT and AQLQ scores were significantly higher in ATA patients with ICSLABA than with ICS-LABA plus LTRA (ACT: $21.77 \pm$ 3.18 vs $19.84 \pm 4.26, P=0.041$,AQLQ: $186.96 \pm 23.61$ vs $157.23 \pm 40.63 ; P<0.001$ for both). The ACQ score showed no significant difference $(4.680 \pm 6.04$ vs $6.97 \pm$ 5.53, $P=0.092$ ) between the 2 groups. Regarding the comorbidities, chronic rhinosinusitis was more common in AERD than in ATA patients (40 [85.1\%] in AERD and $35[38.9 \%]$ in ATA patients, $P<0.001)$. There were no significant differences in prevalence of gastroesophageal reflux disease and obesity between patients with AERD and those with ATA (4 [8.5\%] vs 7 [7.8\%], $P=0.881 ; 12[25.5]$ vs $32[35.6], P=0.254)$.

\section{Serum and Urine Levels of $\mathrm{LTE}_{4}, \mathrm{I5}$ - HETE, TXB ${ }_{2}$ and $\mathrm{PGF}_{2} \alpha$ in the Study Subjects}

In $\mathrm{HCs}$, the serum levels of $\mathrm{LTE}_{4}, 15$-HETE, $\mathrm{TXB}_{2}, \mathrm{PGF}_{2}$ $\alpha$ and $\mathrm{LTE}_{4} / \mathrm{PGF}_{2} \alpha$ ratio were found to be $0.03 \pm 0.06 \mathrm{ng} /$ $\mathrm{mL}, 0.97 \pm 0.97 \mathrm{ng} / \mathrm{mL}, 0.26 \pm 0.70 \mathrm{ng} / \mathrm{mL}, 0.07 \pm 0.15$ $\mathrm{ng} / \mathrm{mL}$ and $0.09 \pm 0.19$ (mean $\pm \mathrm{SD}$ ), respectively; the urine levels of $\mathrm{LTE}_{4}, 15-\mathrm{HETE}, \mathrm{TXB}_{2}, \mathrm{PGF}_{2} \alpha$ and $\mathrm{LTE}_{4}$ $/ \mathrm{PGF}_{2} \alpha$ ratio were $469.11 \pm 1390.33 \mathrm{pg} / \mathrm{mg}$ creatinine, $412.06 \pm 1423.53 \mathrm{pg} / \mathrm{mg}$ creatinine, $381.92 \pm 703.50 \mathrm{pg} /$ $\mathrm{mg}$ creatinine, $781.11 \pm 454.50 \mathrm{pg} / \mathrm{mg}$ creatinine and 0.22 \pm 0.48 (Figure 2).

The serum levels of $\mathrm{LTE}_{4}, \mathrm{LTE}_{4} / \mathrm{PGF}_{2} \alpha$ ratio and the urine levels of $\mathrm{LTE}_{4}, \mathrm{PGF}_{2} \alpha$ and $\mathrm{LTE}_{4} / \mathrm{PGF}_{2} \alpha$ ratio showed significantly different among groups of AERD, ATA, and HC (ANOVA, $P=0.014, P=0.016, P=0.007, P=0.010$, and $P<0.001$, respectively) (Figure 2). The urine levels of $\mathrm{LTE}_{4}$, and the serum and urine levels of $\mathrm{LTE}_{4} / \mathrm{PGF}_{2} \alpha$ ratio were significantly higher in the AERD group than in the ATA group (urine: $\mathrm{LTE}_{4}, 539.25 \pm 789.53 \mathrm{pg} / \mathrm{mg}$ creatinine vs $161.57 \pm 328.94 \mathrm{pg} / \mathrm{mg}$ creatinine, serum: $\mathrm{LTE}_{4} / \mathrm{PGF}_{2} \alpha$, $0.62 \pm 0.81$ vs $0.39 \pm 0.46$, urine: $\mathrm{LTE}_{4} / \mathrm{PGF}_{2} \alpha, 0.39 \pm$ 0.46 vs $0.12 \pm 0.21 ; P<0.001, P=0.009$ and $P<0.001$, respectively), while marginal differences were noted in the serum levels of $\mathrm{TXB}_{2}$; no significant differences were noted in the levels of 15-HETE (Figure 2). Subsequently, we further analyzed the association of the levels of $\mathrm{LTE}_{4}$ and $\mathrm{LTE}_{4}$ $/ \mathrm{PGF}_{2 \alpha}$ ratio with clinical parameters. Receiver operating characteristic analysis (Figure S1) revealed that the urine levels of $\mathrm{LTE}_{4}$ and $\mathrm{LTE}_{4} / \mathrm{PGF}_{2 \alpha}$ ratio could discriminate AERD patients from ATA patients $(P<0.001$, AUC $=0.746$ 


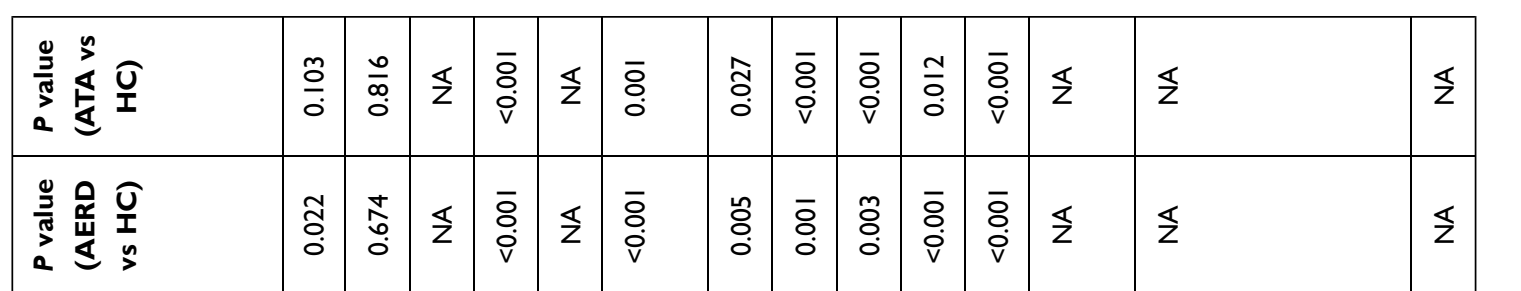

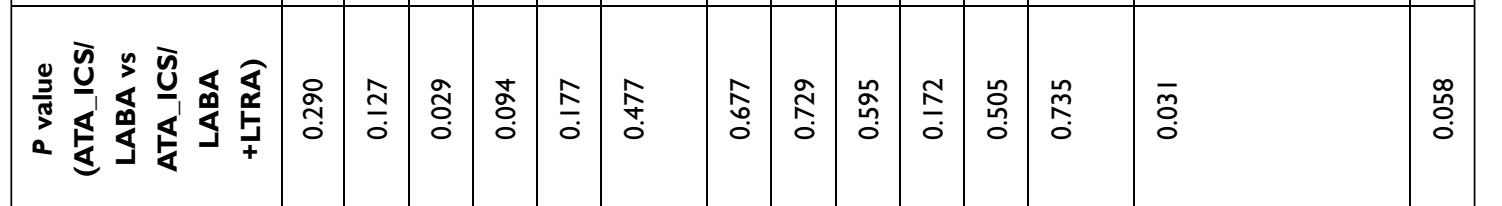

\begin{tabular}{|c|c|c|c|c|c|c|c|c|c|c|c|}
\hline 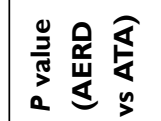 & 愛总 & & :ُ & 言 & $\frac{\frac{2}{0}}{0}$ & & & & 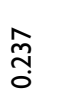 & 兽 & 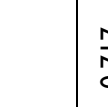 \\
\hline 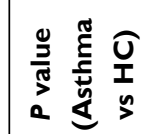 & 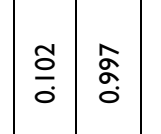 & $\begin{array}{ll}\bar{z} \\
z \\
\bar{O}\end{array}$ & $\overline{\frac{5}{y}} \frac{1}{z}$ & & : & & & & $\underline{z}$ & $\frac{\underline{z}}{z}$ & 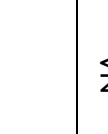 \\
\hline
\end{tabular}

\begin{tabular}{|c|c|c|c|c|c|c|c|c|c|c|c|c|c|}
\hline 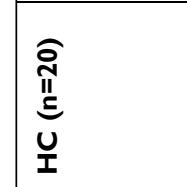 & & & 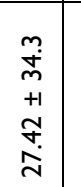 & 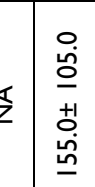 & & & 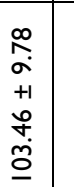 & & $z$ & $\frac{s}{z}$ & & & $\frac{1}{z}$ \\
\hline 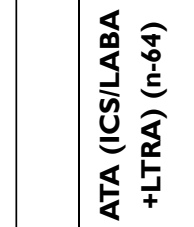 & 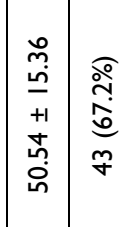 & & 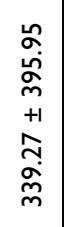 & 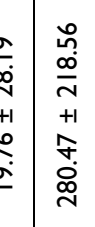 & & & 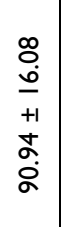 & & 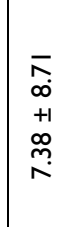 & & & & 8 \\
\hline
\end{tabular}

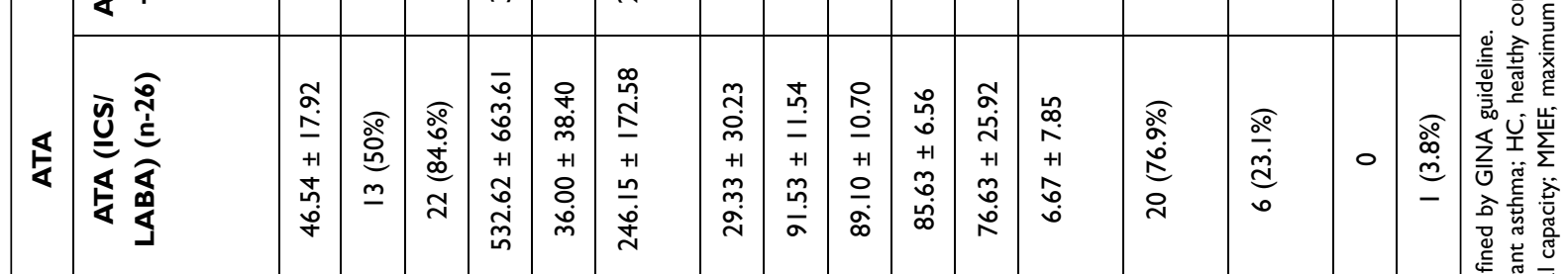

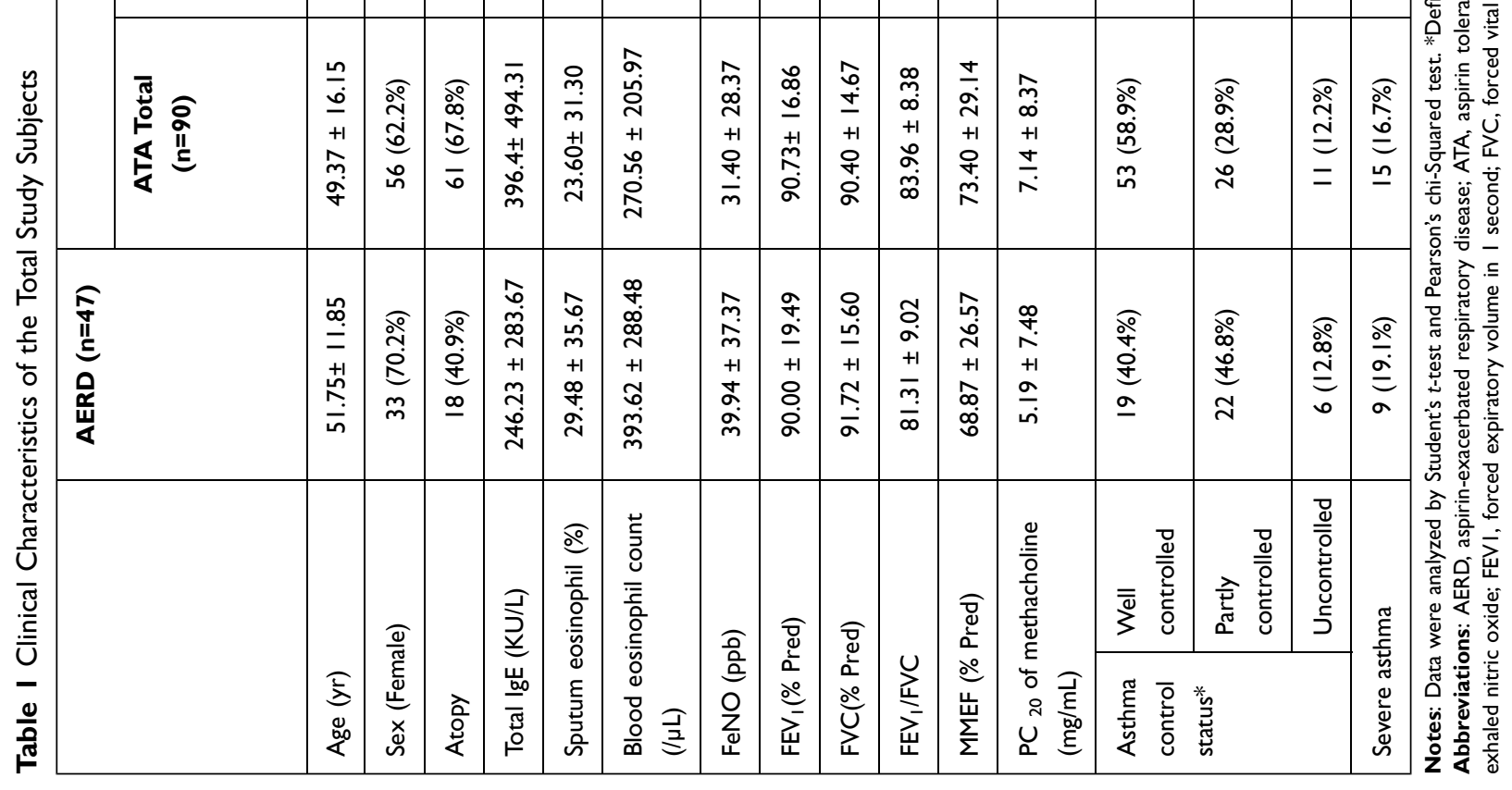



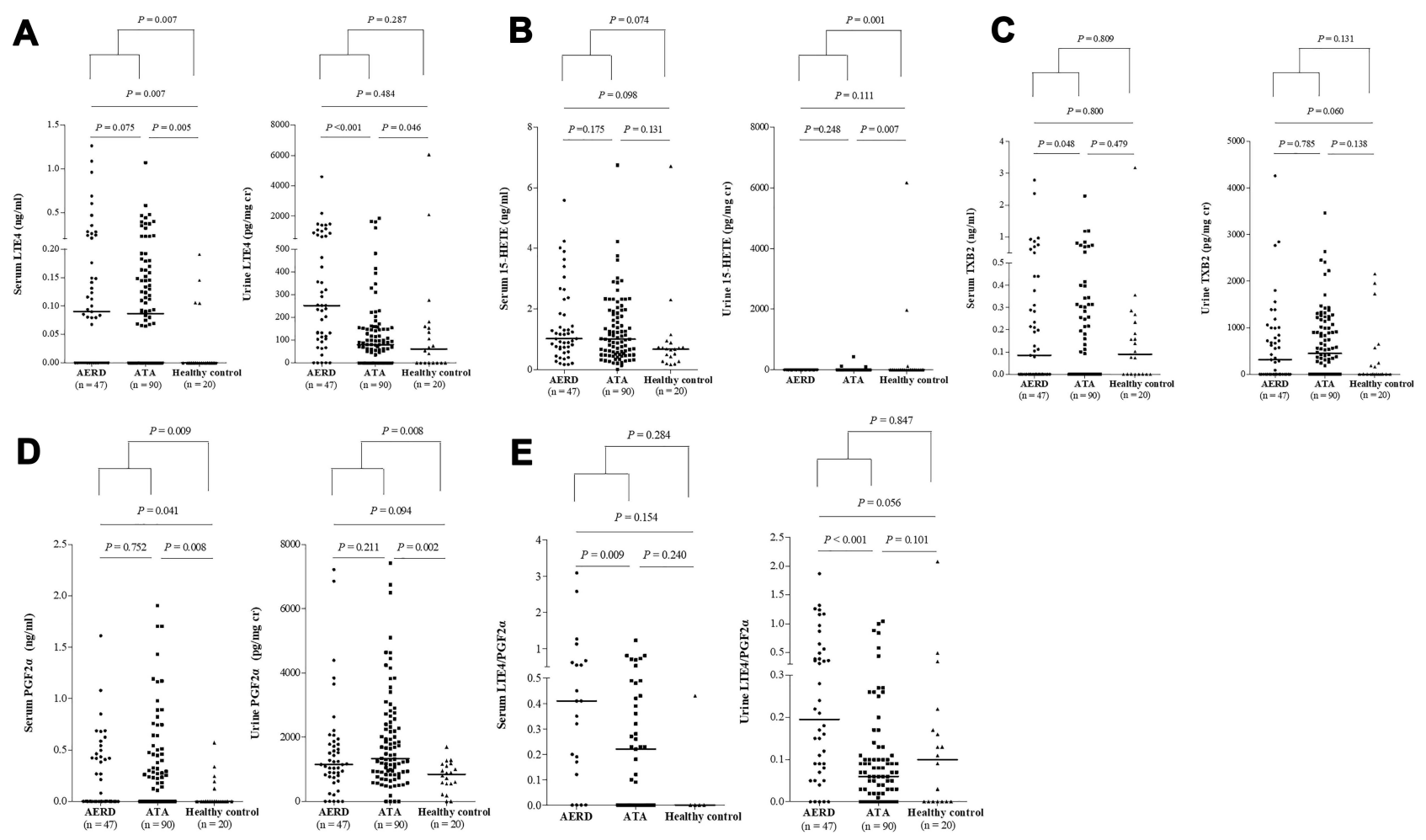

Figure 2 The serum and urine levels of lipid mediators in the 3 study groups. (A) LTE 4 . (B) I5-HETE. (C) TXB T $_{2}$ (D) PGF 2 . (E) LTE $_{4} /$ PGF $_{2} \alpha$. Note: General linear regression analysis was performed to compare metabolite levels between the groups.

Abbreviations: AERD, aspirin-exacerbated respiratory disease; ATA, aspirin tolerant asthma; LT, leukotriene; HETE, hydroxyeicosatetraenoic acid; TXB ${ }_{2}$, II-dehydro thromboxane B2; PG, prostaglandin.

and $P<0.001$, AUC $=0.747$, respectively). Significant positive correlations were observed between the serum and urine levels of $\mathrm{LTE}_{4}$, but no correlation was found between the serum and urine levels of other metabolites ( Figure S2).

\section{Serum and Urine Levels of $\mathrm{LTE}_{4}$ in Asthmatics According to LTRA Medication}

The serum and urine levels of $\mathrm{LTE}_{4}$ and $\mathrm{LTE}_{4} / \mathrm{PGF}_{2 \alpha}$ ratio were found to be $0.15 \pm 0.23 \mathrm{ng} / \mathrm{mL}, 0.43 \pm 0.59,324.44 \pm$ $600.14 \mathrm{pg} / \mathrm{mg}$ creatinine and $0.23 \pm 0.36$ in asthmatics who had been taking ICS/LABA plus LTRA. The serum levels of $\mathrm{LTE}_{4} / \mathrm{PGF}_{2} \alpha$ ratio and the urine levels of $\mathrm{LTE}_{4}$, and $\mathrm{LTE}_{4} / \mathrm{PGF}_{2} \alpha$ ratio showed significantly different among groups (ANOVA, $P=0.031, \quad P=0.001$, and $P<0.001$, respectively) as shown in Figure 3. The urine levels of $\mathrm{LTE}_{4}$, and the serum and urine levels of $\mathrm{LTE}_{4}$ $/ \mathrm{PGF}_{2 \alpha}$ ratio were significantly higher in the AERD patients than in the ATA patients who were on LTRA medication ( $P=0.001, P=0.023$ and $P<0.001$, respectively) (Figure 3A and B). Neither serum nor urine levels of $\mathrm{LTE}_{4}$ or $\mathrm{LTE}_{4} / \mathrm{PGF}_{2 \alpha}$ ratio showed any significant differences in the ATA group between ATA patients on ICS-LABA and on ICS-LABA plus LTRA ( $P>0.05$ for all). Considering the higher proportion of well-controlled asthmatics in the ATA group with ICS-LABA use, multivariate linear regression analysis was performed to adjust for asthma control status. After adjusting for asthma control status (regardless of GINA guideline asthma control status, ACT, ACQ and AQLQ score), neither serum or urine levels of $\mathrm{LTE}_{4}$ or $\mathrm{LTE}_{4} / \mathrm{PGF}_{2} \alpha$ ratio showed any significant differences according to LTRA medication in ATA patients $(P>0.05$ for all). There were no differences in the serum or urine levels of 15-HETE, $\mathrm{TXB}_{2}$ or $\mathrm{PGF}_{2 \alpha}$ between the AERD and ATA groups according to asthma control status or clinical parameters.

\section{Serum and Urine $\mathrm{LTE}_{4}$ Levels in Asthmatics According to Asthma Control Status and Severity}

Although the serum and urine levels of $\mathrm{LTE}_{4}$ and $\mathrm{LTE}_{4}$ $/ \mathrm{PGF}_{2} \alpha$ ratio were not significantly different by ANOVA, uncontrolled AERD patients showed significantly higher serum and urine levels of $\mathrm{LTE}_{4}$ compared to controlled or 

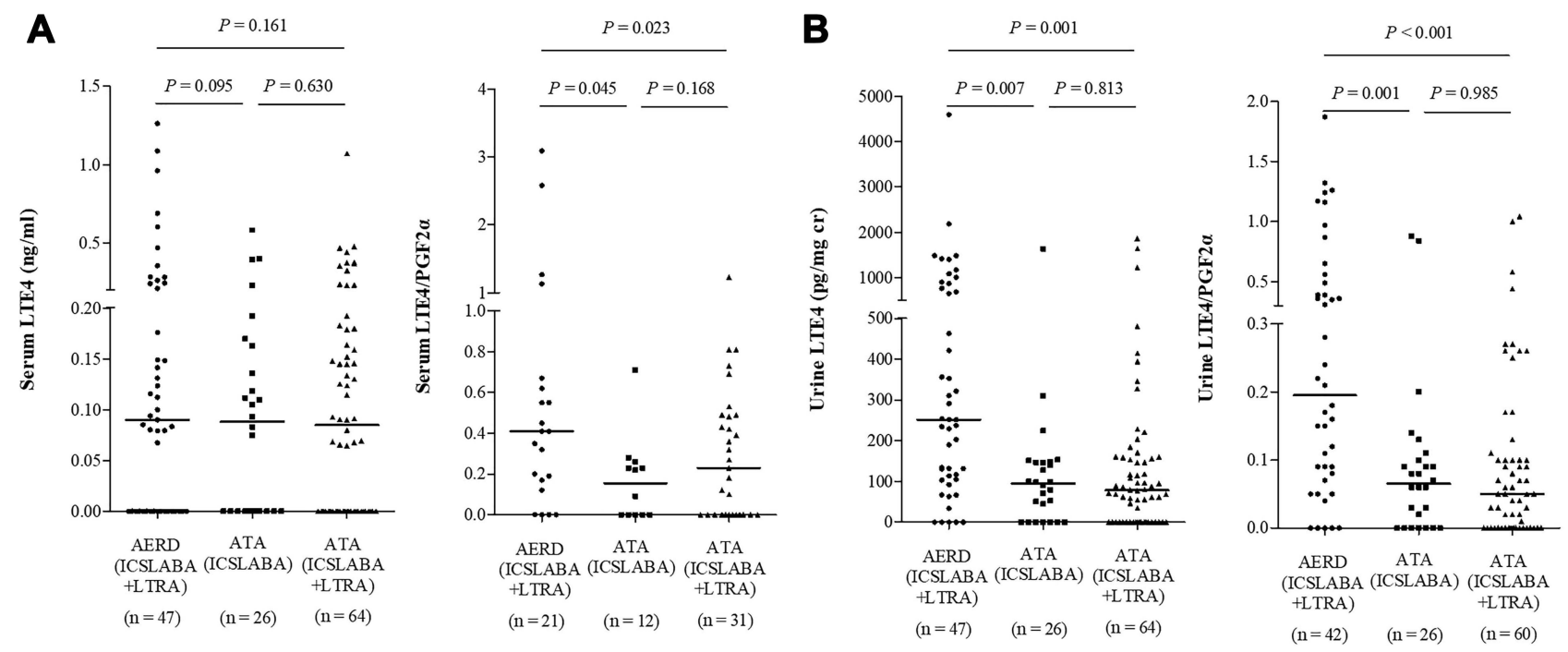

Figure 3 The serum $(\mathbf{A})$ and urine $(\mathbf{B})$ levels of $\mathrm{LTE}_{4}$ in asthmatics according to LTRA medication.

Note: General linear regression analysis was performed to compare metabolite levels between the groups.

Abbreviations: AERD, aspirin-exacerbated respiratory disease; ATA, aspirin tolerant asthma; LT, leukotriene; PG, prostaglandin; ICSLABA, inhaled corticosteroid/longacting beta agonist; LTRA, leukotriene receptor antagonist.

partly controlled AERD patients (Figure 4A). The serum levels of 15-HETE and $\mathrm{TXB}_{2}$ were significantly higher in uncontrolled AERD patients than in controlled or partly controlled patients $(P=0.002$ and $P=0.011$, respectively), while no differences were noted in the serum levels of $\mathrm{PGF}_{2 \alpha}$ (data not shown). When asthma control status was classified according to ACQ scores, the AERD patients with ACQ $\geq 1.5$ showed significantly higher serum levels of $\mathrm{LTE}_{4}$ and $\mathrm{LTE}_{4} / \mathrm{PGF}_{2 \alpha}$ ratio compared to controlled AERD patients $(P=0.025$ and $P=0.011$, respectively $)$ (Figure S3).

No differences in the serum or urine levels of $\mathrm{LTE}_{4}$ or $\mathrm{LTE}_{4} / \mathrm{PGF}_{2 \alpha}$ ratio were observed in ATA patients according to asthma control status as defined by the GINA guideline, ACT $\leq 20$ or ACQ-6 $\geq 1.5$ (Figure 4B). However, ACQ scores significantly associated with the urine levels of $\mathrm{LTE}_{4}$ after adjusting for age and sex using multivariate linear regression analysis in ATA patients $(P=0.032, \mathrm{~B}=11.931, \operatorname{Exp}[\mathrm{B}]=151,979.07)$.

There were no significant differences in the serum or urine levels of $\mathrm{LTE}_{4}, 15-\mathrm{HETE}, \mathrm{TXB}_{2} \mathrm{PGF}_{2 \alpha}$ or $\mathrm{LTE}_{4}$ $/ \mathrm{PGF}_{2} \alpha$ ratio between non-severe asthma and severe asthma (data not shown).

\section{Correlation Between $\mathrm{LTE}_{4}$ Levels and Type 2 Inflammatory Markers}

The serum and urine levels of $\mathrm{LTE}_{4}$ and $\mathrm{LTE}_{4} / \mathrm{PGF}_{2} \alpha$ ratio were found to be significantly higher in patients with blood eosinophil counts $\geq 300 / \mu \mathrm{L}$ (serum, $P=0.001$ and
$P=0.020$ respectively; urine, $P<0.001$ for each) (Figure 5). There were weak but significant positive associations of the serum and urine levels of $\mathrm{LTE}_{4}$ with blood eosinophil counts and FeNO levels (serum: $r=0.426$, $P<0.001$, and $r=0.243, P=0.005$, respectively; urine: $r=0.386, P<0.001$, and $r=0.356, P<0.001$, respectively) (Figure 6A), These correlations were seen in both AERD and ATA patients (AERD: serum, $r=0.446, P=0.002$, and $P=0.168$; urine, $r=0.389, P=0.007$ and $r=0.524, P<0.001$; ATA: serum, $r=0.367, P<0.001$, and $r=0.263, P=0.014$; urine, $r=0.274, P=0.009$ and $P=0.566$, respectively).

Patients with chronic rhinosinusitis (CRS) had significantly higher serum and urine levels of $\mathrm{LTE}_{4}$ and $\mathrm{LTE}_{4}$ $/ \mathrm{PGF}_{2 \alpha}$ ratio than those without (serum: $P=0.001$ and $P=0.018$, respectively; urine: $P=0.001$ and $P<0.001$, respectively) (data not shown). No significant associations were found between the serum and urine levels of $\mathrm{LTE}_{4}$ and lung function parameters, including $\mathrm{FEV}_{1}, \mathrm{FVC}$ and MMEF (Figure 6B), in all asthmatics.

However, when study subjects were classified into the high ( $\geq$ the median level of $\left.\mathrm{LTE}_{4}\right)$ vs low $(<$ the median level of $\left.\mathrm{LTE}_{4}\right)_{\mathrm{LTE}_{4}}$ groups, significantly lower predicted values of $\mathrm{FEV}_{1}, \mathrm{FVC}$ and MMEF were noted in subjects with high $\mathrm{LTE}_{4}$ within the AERD group $(P=0.006$, $P=0.015$ and $P=0.016$, respectively) (Tables $\mathrm{S} 1$ and $\underline{\mathrm{S} 2}$ ). Also, blood eosinophil counts were significantly higher in the high $\mathrm{LTE}_{4}$ group than in the low $\mathrm{LTE}_{4}$ group $(P<0.05)$. In addition, to investigate factors affecting the serum and urine levels of $\mathrm{LTE}_{4}$, multivariate analyses of aspirin 

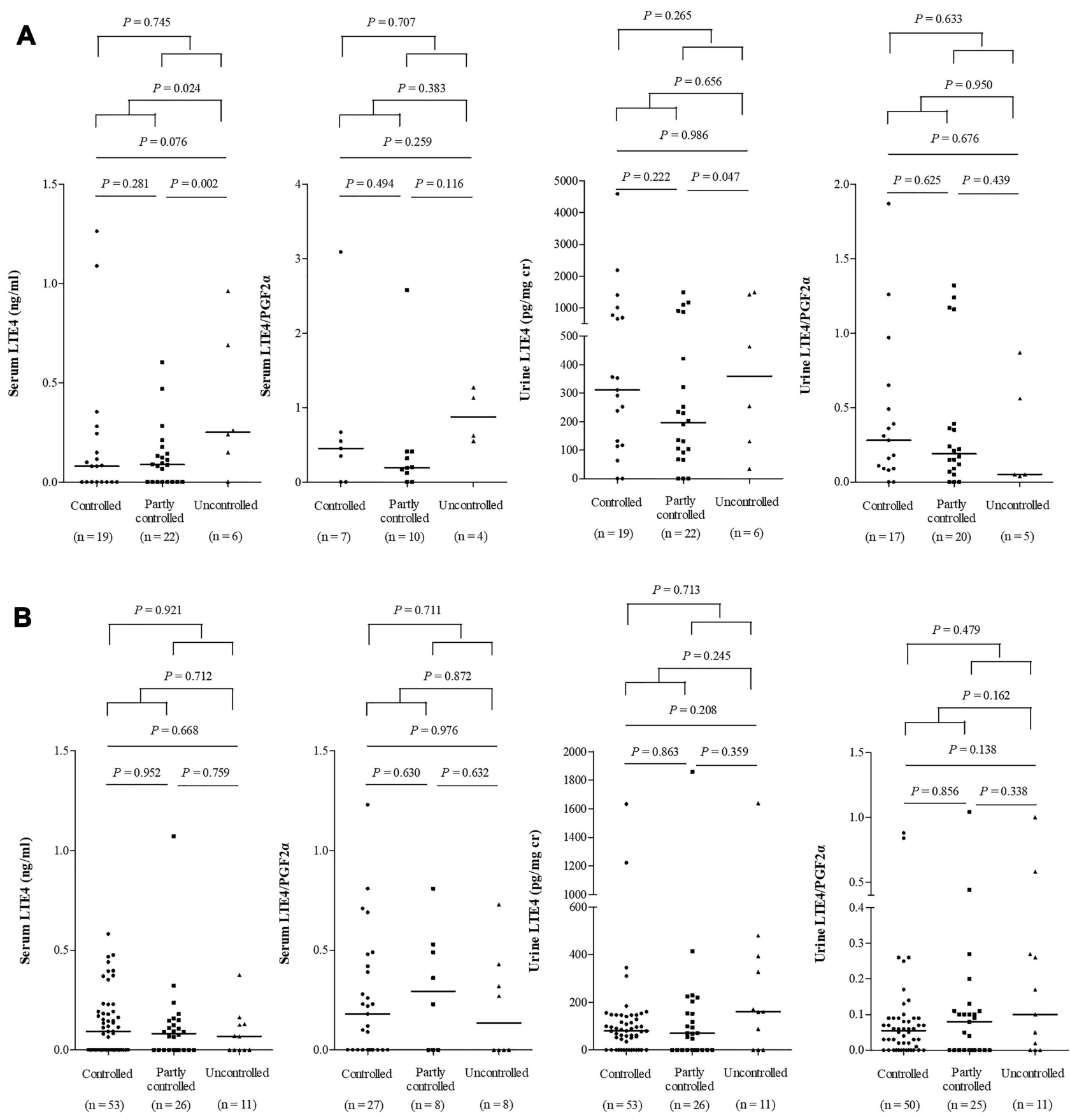

Figure 4 The levels of $\mathrm{LTE}_{4}$ in patients according to asthma control status in AERD (A) and ATA (B) patients.

Notes: Asthma control status was defined by GINA guideline. General linear regression analysis was performed to compare metabolite levels between the groups. Abbreviations: AERD, aspirin-exacerbated respiratory disease; ATA, aspirin tolerant asthma; LT, leukotriene; PG, prostaglandin.

sensitivity, presence of CRS, asthma control status by the GINA guideline, blood eosinophil counts, FeNO levels age and sex were performed. Blood eosinophil count was the only factor significantly affecting the serum levels of $\mathrm{LTE}_{4}(P<0.001, \mathrm{~B}=0.000336, \operatorname{Exp}[\mathrm{B}]=1.000336)$ (Table $\underline{\mathrm{S} 3})$. Aspirin sensitivity, blood eosinophil counts and FeNO levels significantly affected the urine levels of
$\mathrm{LTE}_{4} \quad(P=0.009, \quad \mathrm{~B}=257.246, \quad \operatorname{Exp}[\mathrm{B}]=5.26 \mathrm{E}+111 ;$ $P<0.001, \quad \mathrm{~B}=0.674, \quad \operatorname{Exp}[\mathrm{B}]=1.961, \quad$ and $P=0.001$, $\mathrm{B}=4.444, \operatorname{Exp}[\mathrm{B}]=85.094$, respectively) (Table $\mathrm{S} 4$ ).

\section{Discussion}

This is the first study to demonstrate that $\mathrm{LTE}_{4}$ levels remain high and show a strong association with blood 


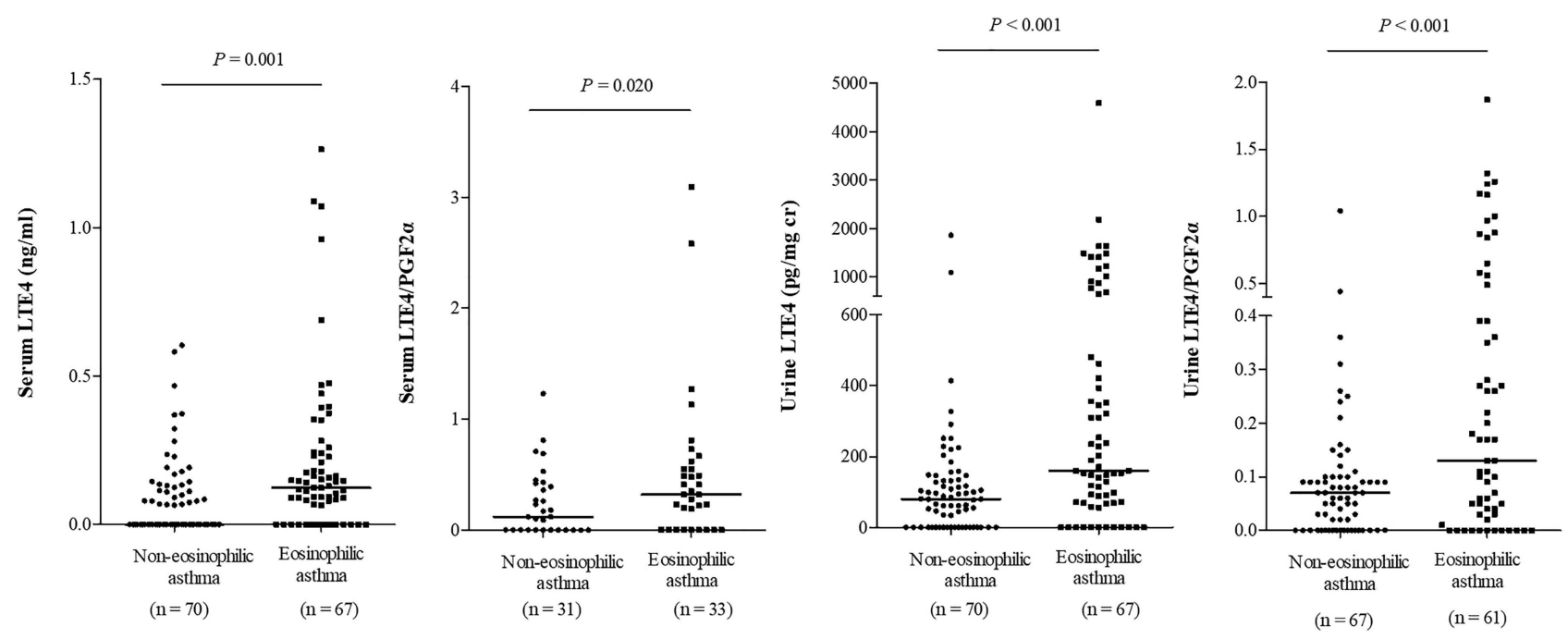

Figure 5 The levels of $\mathrm{LTE}_{4}$ in patients with eosinophilic asthma and non-eosinophilic asthma.

Notes: Eosinophilic asthma was defined when the blood eosinophil count $\geq 300 / \mu \mathrm{L}$. General linear regression analysis was performed to compare metabolite levels between the groups.

Abbreviations: LT, leukotriene; PG, prostaglandin.
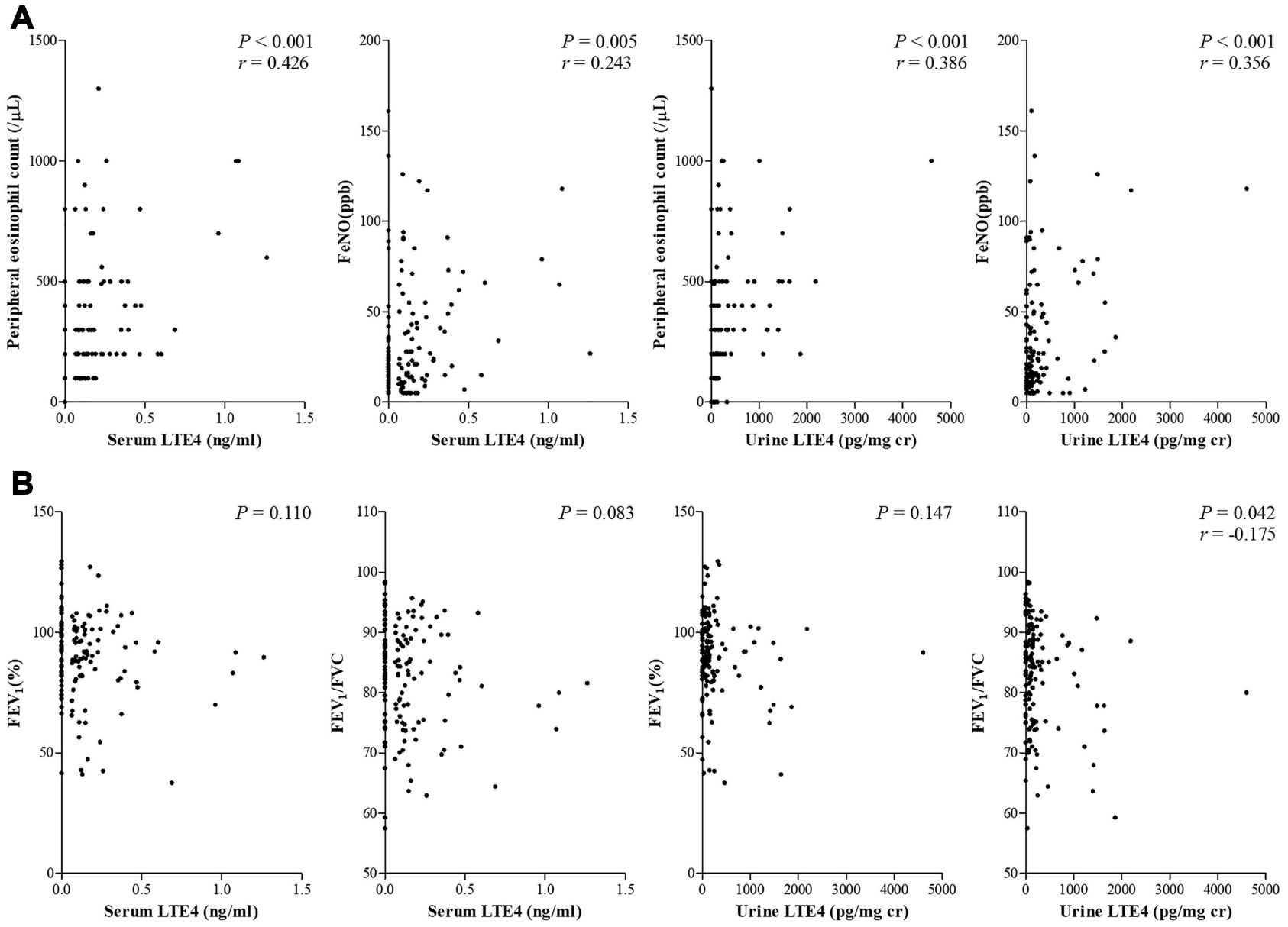

Figure 6 Correlation between the levels of $\mathrm{LTE}_{4}$ and clinical parameters $((\mathbf{A})$ eosinophil markers, $(\mathbf{B})$ pulmonary function).

Note: $P$ values were obtained by Pearson's correlation test.

Abbreviations: LT, leukotriene; FeNO, fractional exhaled nitric oxide; FEVI, forced expiratory volume in I second; FVC, forced vital capacity. 
eosinophil counts, FeNO levels and asthma control status in asthmatic patients despite maintenance medications with ICS-LABA plus LTRA. First, we showed that, in a subpopulation of AERD patients with high $\mathrm{LTE}_{4}$ levels, airway inflammation could not be fully suppressed by maintenance medications according to current treatment guidelines, ie ICS-LABA plus LTRA, suggesting that CysLTs-mediated inflammation does not solely drive disease severity in these patients. Secondly, high $\mathrm{LTE}_{4}$ levels in ATA patients did not show association with lung function, and the proportion of well-controlled patients was lower in patients treated with ICS-LABA plus LTRA when compared to those treated with ICS-LABA alone. Thirdly, in both AERD and ATA patients, high $\mathrm{LTE}_{4}$ levels were linked with persistent eosinophilia. Based on these findings, we propose that earlier eosinophilia-targeting treatment could be a better option than LTRA when a combination of ICS-LABA does not lead to disease control.

ICS-LABA is known to be the mainstay of treatment options for asthma. ${ }^{13}$ LTRA provides an additive benefit for asthmatics whose asthma is not adequately controlled with ICS alone or ICS-LABA, which is derived from the limited capability of corticosteroids to inhibit the leukotriene synthesis pathway. ${ }^{8,20-23}$ Clinical studies on the efficacy of LTRA treatment have been discrepant, and targeting of the LTRA treatment to asthmatic patients with high $\mathrm{LTE}_{4}$ levels has been suggested to improve the efficacy. The present study performed in a realworld clinical setting clearly demonstrated that the serum and urine levels of $\mathrm{LTE}_{4}$ were high in a considerable number of adult asthmatics, and the higher in the AERD group than in the ATA group despite ICSLABA plus LTRA medications, suggesting that LTRA medication does not modulate the levels of $\mathrm{LTE}_{4}{ }^{5}$ LTRA, a selective antagonist of the CysLT $_{1}$ receptor, is known to directly block the action of CysLTs, but not their synthesis. However, it has been speculated that the synthesis of CysLTs could be reduced in the chronic use of LTRAs. ${ }^{24} \mathrm{LTD}_{4}$ up-regulates IL-13 and its receptor, and IL-13 production up-regulates the synthesis of CysLTs and then the expression of the CysLT $_{1}$ receptor. $^{25}$ Furthermore, the anti-inflammatory activity of LTRAs could decrease the influx of inflammatory cells in the lungs. Accordingly, the cross-talk between CysLTs and type 2 cytokines could result in the selfperpetuating circuit of airway inflammation. ${ }^{20}$ Blocking of $\mathrm{CysLT}_{1}$ receptor could interrupt this and secondarily down-regulate the levels of $\mathrm{LTE}_{4}$. However, this kind of modulation of the levels of CysLTs by LTRA was not supported by our findings.

Human eosinophils are the main source of CysLTs, and CysLTs exert autocrine signals regulating IL-4 secretion and activating eosinophils. ${ }^{26}$ Recent studies suggest that CysLTs also induce innate immune responses via stimulating release of thymic stromal lymphopoietin (TSLP) and IL-33 from type 2 alveolar cells and epithelial cells. ${ }^{14,27,28}$ Therefore, TSLP/IL-33 and CysLTs synergistically activate group 2 innate lymphoid cells, provoking IL-5 release and persistent eosinophilic inflammation. ${ }^{29}$ The present study showed that there were significant associations between the serum/urine levels of $\mathrm{LTE}_{4}$ and blood eosinophil counts/FeNO levels, with a negative correlation between uLTE4 and FEV1/FVC, indicating a close link between $\mathrm{LTE}_{4}$ and type 2/eosinophilic airway inflammation.

The strength of the present study is that this study was performed in a real-world clinical setting, which also has its limitations in design. Although the critical factors that may affect the levels of $\mathrm{LTE}_{4}$ were controlled (eg asthmatics who used biologics or showed recent asthma exacerbation were excluded, LTRA users were separately analyzed), other factors that could affect the levels of $\mathrm{LTE}_{4}$ including medications for comorbidities, ICS adherence or diet pattern were not completely controlled. Because of the cross-sectional study design, further studies are needed to confirm our results. Altogether, our findings support earlier reports that a subgroup of patients with asthma, both in the ATA and AERD groups, is not adequately controlled with a combination of ICS-LABA plus LTRA. ${ }^{30}$ In addition, targeting LTRA treatment to patients with high $\mathrm{LTE}_{4}$ levels does not necessarily improve the efficacy, since persistent eosinophilia, lung inflammation and progressive lung function decline were seen in patients with high $\mathrm{LTE}_{4}$ despite LTRA treatment along with ICS-LABA. Another plausible explanation for the poor efficacy of LTRA treatment may be that an unidentified $\mathrm{LTE}_{4}$ receptor could mediate airway inflammation in asthma and resist currently available LTRAs. ${ }^{31,32}$ However, no such recent evidence exists to support this interpretation.

In conclusion, our results emphasize the need for other therapeutic options in asthmatic patients with high $\mathrm{LTE}_{4}$ levels, such as targeting eosinophils, TSLP or IL-33, and suggest the role of CysLTs as a major driver of eosinophilic airway inflammation and remodeling. ${ }^{33}$ 


\section{Abbreviations}

AA, arachidonic acid; ACQ, asthma control questionnaire; $\mathrm{ACT}$, asthma control test; AERD, aspirin-exacerbated respiratory disease; AQLQ, asthma quality of life; ATA, aspirintolerant asthma; CysLTs, Cysteinyl leukotrienes; FeNO, Fractional exhaled NO; GINA, Global Initiative for Asthma guideline; IgE, immunoglobulin E; ICS, inhaled corticosteroid; LABA, long-acting $\beta_{2}$-agonist; LC-MS/MS, liquid chromatography-tandem mass spectrometry; LTRA, leukotriene receptor antagonist; Lys-ASA BPT, lysine-aspirin bronchial provocation test; PGF2 $\alpha$, prostaglandin F2 $\alpha$; TSLP, thymic stromal lymphopoietin; TXB2, 11-dehydro thromboxane B2; 15-HETE, 15-hydroxyeicosatetraenoic acid.

\section{Acknowledgments}

We thank Fanyi Jiang, Katerina Pardali, Outi Vaarala and Joo-Youn Cho for their cooperation and contribution to this research.

\section{Author Contributions}

All authors made a significant contribution to the work reported, whether that is in the conception, study design, execution, acquisition of data, analysis and interpretation, or in all these areas; took part in drafting, revising or critically reviewing the article; gave final approval of the version to be published; have agreed on the journal to which the article has been submitted; and agree to be accountable for all aspects of the work.

\section{Funding}

This study was supported by AstraZeneca, a grant from the Korean Health Technology R\&D Project, Ministry of Health \& Welfare, Republic of Korea (HR16C0001) and the National Research Foundation of Korea (NRF) grant funded by the Korea government (MSIP) (No: 2020R1I1A3051800).

\section{Disclosure}

The authors declare that they have no competing interests.

\section{References}

1. Israel E, Reddel HK. Severe and difficult-to-treat asthma in adults. $N$ Engl J Med. 2017;377(10):965-976. doi:10.1056/NEJMra1608969

2. Fajt ML, Wenzel SE. Development of new therapies for severe asthma. Allergy Asthma Immunol Res. 2017;9(1):3-14. doi:10.4168/ aair.2017.9.1.3

3. Holgate ST. The sentinel role of the airway epithelium in asthma pathogenesis. Immunol Rev. 2011;242(1):205-219. doi:10.1111/ j.1600-065X.2011.01030.x
4. Lee JH, Jung CG, Park HS. An update on the management of aspirin-exacerbated respiratory disease. Expert Rev Respir Med. 2018;12(2):137-143. doi:10.1080/17476348.2018.1417843

5. Ban GY, Cho K, Kim SH, et al. Metabolomic analysis identifies potential diagnostic biomarkers for aspirin-exacerbated respiratory disease. Clin Exp Allergy. 2017;47(1):37-47. doi:10.1111/cea.12797

6. Gauvreau GM, Parameswaran KN, Watson RM, O’byrne PM. Inhaled leukotriene E4, but not leukotriene D4, increased airway inflammatory cells in subjects with atopic asthma. Am J Respir Crit Care Med. 2001;164 (8):1495-1500. doi:10.1164/ajrccm.164.8.2102033

7. Green S, Malice M, Tanaka W, Tozzi C, Reiss T. Increase in urinary leukotriene LTE4 levels in acute asthma: correlation with airflow limitation. Thorax. 2004;59(2):100-104. doi:10.1136/thorax.2003.006825

8. Reiss TF, Dockhorn RJ, Shingo S, Seidenberg B, Edwards TB; for the Montelukast Clinical Research Study Group. Montelukast, a once-daily leukotriene receptor antagonist, in the treatment of chronic asthma. Arch Intern Med. 1998;158:1213-1220. doi:10.1001/ archinte.158.11.1213

9. Hallstrand TS, Henderson WR. An update on the role of leukotrienes in asthma. Curr Opin Allergy Clin Immunol. 2010;10(1):60-66. doi:10.1097/ACI.0b013e32833489c3

10. Bosse Y, Thompson C, McMahon S, Dubois C, Stankova J, RolaPleszczynski M. Leukotriene D4-induced, epithelial cell-derived transforming growth factor $\beta 1$ in human bronchial smooth muscle cell proliferation. Clin Exp Allergy. 2008;38(1):113-121.

11. Altraja S, Jaama J, Altraja A. Proteome changes of human bronchial epithelial cells in response to pro-inflammatory mediator leukotriene E4 and pro-remodelling factor TGF- $\beta 1$. J Proteomics. 2010;73 (6):1230-1240. doi:10.1016/j.jprot.2010.02.017

12. Montuschi P, Peters-Golden ML. Leukotriene modifiers for asthma treatment. Clin Exp Allergy. 2010;40(12):1732-1741. doi:10.1111/ j.1365-2222.2010.03630.x

13. Global strategy for asthma management and prevention; 2020 [cited July 10, 2020]. Available from: http://ginasthma.org/gina-reports/. Accessed October 5, 2021.

14. Woo SD, Luu QQ, Park HS. NSAID-exacerbated respiratory disease (NERD): from pathogenesis to improved care. Front Pharmacol. 2020;11:1147. doi:10.3389/fphar.2020.01147

15. Park H. Early and late onset asthmatic responses following lysineaspirin inhalation in aspirin-sensitive asthmatic patients. Clin Exp Allergy. 1995;25(1):38-40. doi:10.1111/j.1365-2222.1995.tb01000.x

16. Crimi C, Campisi R, Noto A, et al. Comparability of asthma control test scores between self and physician-administered test. Respir Med. 2020;170:106015. doi:10.1016/j.rmed.2020.106015

17. Juniper EF, Bousquet J, Abetz L, Bateman ED; Goal Committee. Identifying 'well-controlled' and 'not well-controlled' asthma using the Asthma Control Questionnaire. Respir Med. 2006;100 (4):616-621. doi:10.1016/j.rmed.2005.08.012

18. Chung KF, Wenzel SE, Brozek JL, et al. International ERS/ATS guidelines on definition, evaluation and treatment of severe asthma. Eur Respir J. 2014;43(2):343-373. doi:10.1183/09031936.00202013

19. Ban GY, Youn DY, Ye YM, Park HS. Increased expression of serine palmitoyl transferase and ORMDL3 polymorphism are associated with eosinophilic inflammation and airflow limitation in aspirin-exacerbated respiratory disease. PLoS One. 2020;15(10): e0240334. doi:10.1371/journal.pone.0240334

20. Peters-Golden M, Henderson WR. Leukotrienes. $N$ Engl J Med. 2007;357(18):1841-1854. doi:10.1056/NEJMra071371

21. Sandrini A, Ferreira IM, Gutierrez C, Jardim JR, Zamel N, Chapman KR. Effect of montelukast on exhaled nitric oxide and nonvolatile markers of inflammation in mild asthma. Chest. 2003;124(4):1334-1340. doi:10.1378/chest.124.4.1334

22. Ilowite J, Webb R, Friedman B, et al. Addition of montelukast or salmeterol to fluticasone for protection against asthma attacks: a randomized, double-blind, multicenter study. Ann Allergy Asthma Immunol. 2004;92(6):641-648. doi:10.1016/S1081-1206(10)61430-5 
23. Laviolette M, Malmstrom K, Lu S, et al. Montelukast added to inhaled beclomethasone in treatment of asthma. Am J Respir Crit Care Med. 1999;160(6):1862-1868. doi:10.1164/ajrccm.160.6.9803042

24. Cai C, Yang J, Hu S, Zhou M, Guo W. Relationship between urinary cysteinyl leukotriene E4 levels and clinical response to antileukotriene treatment in patients with asthma. Lung. 2007;185(2):105-112. doi:10.1007/s00408-006-0001-8

25. Espinosa K, Bossé Y, Stankova J, Rola-Pleszczynski M. CysLT1 receptor upregulation by TGF- $\beta$ and IL-13 is associated with bronchial smooth muscle cell proliferation in response to LTD4. J Allergy Clin Immunol. 2003;111(5):1032-1040. doi:10.1067/mai.2003.1451

26. Baptista-dos-Reis R, Muniz VS, Neves JS. Multifaceted roles of cysteinyl leukotrienes in eliciting eosinophil granule protein secretion. Biomed Res Int. 2015;2015:848762. doi:10.1155/2015/848762

27. Lund SJ, Portillo A, Cavagnero K, et al. Leukotriene C4 potentiates IL-33-induced group 2 innate lymphoid cell activation and lung inflammation. J Immunol. 2017;199(3):1096-1104. doi:10.4049/ jimmunol.1601569

28. Liu T, Barrett NA, Kanaoka Y, et al. Type 2 cysteinyl leukotriene receptors drive IL-33-dependent type 2 immunopathology and aspirin sensitivity. J Immunol. 2018;200(3):915-927. doi:10.4049/ jimmunol.1700603
29. Jonckheere AC, Bullens DM, Seys SF. Innate lymphoid cells in asthma: pathophysiological insights from murine models to human asthma phenotypes. Curr Opin Allergy Clin Immunol. 2019;19 (1):53-60. doi:10.1097/ACI.0000000000000497

30. Buchheit KM, Laidlaw TM. Update on the management of aspirin-exacerbated respiratory disease. Allergy Asthma Immunol Res. 2016;8(4):298. doi:10.4168/aair.2016.8.4.298

31. Paruchuri S, Tashimo H, Feng C, et al. Leukotriene E4-induced pulmonary inflammation is mediated by the P2Y12 receptor. $J$ Exp Med. 2009;206(11):2543-2555. doi:10.1084/jem.20091240

32. Laidlaw TM, Boyce JA. Cysteinyl leukotriene receptors, old and new; implications for asthma. Clin Exp Allergy. 2012;42 (9):1313-1320. doi:10.1111/j.1365-2222.2012.03982.x

33. Choi Y, Lee Y, Park HS. Which factors associated with activated eosinophils contribute to the pathogenesis of aspirin-exacerbated respiratory disease? Allergy Asthma Immunol Res. 2019;11(3):320. doi:10.4168/aair.2019.11.3.320

\section{Publish your work in this journal}

The Journal of Asthma and Allergy is an international, peer-reviewed open-access journal publishing original research, reports, editorials and commentaries on the following topics: Asthma; Pulmonary physiology; Asthma related clinical health; Clinical immunology and the immunological basis of disease; Pharmacological interventions and new therapies. The manuscript management system is completely online and includes a very quick and fair peer-review system, which is all easy to use. Visit http://www.dovepress.com/testimonials.php to read real quotes from published authors. 